\title{
Modeling Principles, Theory and Methods
}

\author{
Jelenka Savkovic-Stevanovic \\ Faculty of Technology and Metallurgy Belgrade University, Karnegijeva, Belgrade, Serbia
}

\section{Email address:}

stevanoviccace@yahoo.com

\section{To cite this article:}

Jelenka Savkovic-Stevanovic. Modeling Principles, Theory and Methods. Science Research. Vol. 3, No. 3, 2015, pp. 72-78.

doi: 10.11648/j.sr.20150303.15

\begin{abstract}
In this paper modeling theory and methods were developed. Modeling method linking previous knowledge, known facts, scientific laws and hypothesis so that from them can conclude knew knowledge about given domain Integration modeling method was derived. Models in various levels were investigated including variables input and output parameters, and contour conditions. Fundamentals of the microscopic to the macroscopic models were studied. The molecular modeling was considered. The process operation models were derived.
\end{abstract}

Keywords: Method, Integration Modeling, Theory, Elementary Transition, Levels, Integration

\section{Introduction}

In science models have the first of all two purposes logical enabling some conclusions to which can not be come by the other methods, and epistemological to express knowledge and to enable to extent knowledge about real world. The technological advance has addressed the research interest of simulation, supply optimization, in which alternatives were evaluated.

The concomitant advances in, mathematical modeling, numerical methods and measuring systems, bring the new perspectives to investigation. Results of these investigations demonstrate characterization of the equilibrium and non-equilibrium states and transition between them and how it can be used to predict the stability of the system state[1]-[4].

The quantitative approach to modeling and inference involve computing the path coefficients between the variables and using the resulting equation to predict the change in the cause [5]-[7].

In this paper modeling theory, principles and methods were studied.

\section{Theory of Modeling}

Modeling is a phenomena, which is similar to studying phenomena according to definition, and two systems are similar each other, only if exists isomorphism between them. Theory of modeling includes similarity theory.

If there is and only if there similarity of model and phenomena which to examine then principle of modeling is satisfied.

$$
M_{j}\left(x_{i}, y_{i}, z_{i}\right)=M_{j}^{S}
$$

where $M_{j}^{S}$ system model, $x$ is independent variable, $z$ is parameter and is dependent variable $y$.

Variables, parameters and functions describe system state which is modeling. Under mathematical description is understood system of no opposite expressions of variables and parameters which describe system which modeling.

Solution of these expressions are defined in $l$ variant of algorithm $B$ :

$$
M_{j}^{B_{l}}(\ldots .)=M_{j}\left(B_{l}\right)
$$

Proof of similarity modeling theorem is expressed through the minimum deviation of model from actual system.

$$
M_{j}\left(B_{k}\right) \in\left\{\min \mu_{j k}\right\}
$$

where $\mu_{j k}$ is average deviation of model from the actual system.

\section{Modeling Methods}

There two approaches of a system modeling. The first approach is identified with structured knowledge following deductive reasoning, that is, approach which deduced relations on that problem from existing theory [8].

The second approach is identified with post empirical 
knowledge following inductive approach in which model developing from sampling data.

These two approaches represent complementary stages of modeling. The express model is multi-significant, and has significance interpretation of the set values formulas, whose all terms are adequate and significance symbolic representation of some systems.

Mathematical modeling uses symbolic representation by mathematical operators.

The most fundamental description would be based on molecular considerations. The molecular description is distinguished by the fact that it treats an arbitrary system as if it were composed of individual entities, each of which obeys certain rules. Consequently, the properties and state variables of the system are obtained by summing over all of the entities. Quantum mechanics, equilibrium and nonequilibrium statistical mechanics, and classical mechanics would be typical methods of analysis, by which all of the properties and responses of the system could be theoretically calculated.

Molecular modeling of the compound formation is considered. functional groups and their combination.

The structural model is generated according to several levels decomposition of the system unit into phases and components. The modeling begin with system definition. Definition includes all components, input and output attributes, state variables, behavior rules, and initial conditions.

The model generation methodology is a blend of several problem solving paradigms, and hierarchical dynamic goal system construction serves as the basis for a model generation.

Rigorous approach in the microscopic modeling is represented directly by the structure of the elementary transitions referring to the various phenomenological defined processes.

A somewhat less formidable class of model is based on what is the microscopic description. It involves a phenomenological approach and assumes that system can be represented as a continuum. In order words, the detailed molecular interactions are ignored, and certain differential equations are formulated for mass, momentum, and energy.

For non-flow processes or for processes in laminar flow, this stratum of description has many practical uses, although it is often excessively complex. For processes in turbulent flow and with a high degree of mixing, it might attempt to use the statistical theory of turbulence but, as with statistical mechanical concepts, such treatment of a process is not of much practical use, and alternate methods of description are needed.

Since diffusion and reaction transformation are fundamental importance processes, as an illustration and review of the origin and significance of the terms of the basic microscopic balancing, let derive conservation of mass equation for one component in Cartesian coordinates.

$$
\frac{\partial c}{\partial t}=\frac{\partial}{\partial z}\left(D_{l} \frac{\partial c}{\partial z}\right)+S_{r}
$$

where $c$ depends only on height of the system $z$, and time of interval, while $S_{r}$ refers to the net source, $D_{z}$ represents a vertical diffusion coefficient whose values characteristically depend on time of day, height, and location.

In multidimensional scale microscopic level of description is defined by the following equation:

$$
\begin{aligned}
& \frac{\partial c}{\partial t}+v_{x} \frac{\partial c}{\partial x}+v_{y} \frac{\partial c}{\partial y}+v_{z} \frac{\partial c}{\partial z}= \\
& D_{e f}\left(\frac{\partial}{\partial x}\left(\frac{\partial c}{\partial x}\right)+\frac{\partial}{\partial y}\left(\frac{\partial c}{\partial y}+\frac{\partial}{\partial z}\left(\frac{\partial c}{\partial z}\right)+S_{r}\right.\right.
\end{aligned}
$$

where $c$ is concentration described in spatial coordinates as partial differential equation.

$D_{e f}$ is the effective diffusion coefficient, $v_{x}, v_{y}, v_{z}$ denote spatial velocities, and $S_{r}$ is generation term ( chemical reaction, emission, biotransormation, etc.) during the flow.

Multiple gradient description incorporates less detailed information about the internal features of the system of interest than does the microscopic description. The forms of the mathematical equations for this mode of description are suggested by and correspond to the microscopic transport equations with modified coefficients. These coefficients are clearly empirical and must be determined for each type of unit of interest or equipment, although to be fair it should state that with suitable correlations the coefficients obtained under one set of circumstances. The essential features of the multiple gradient is that one or more dispersion terms are important. The multiple gradient balances can be evolved from the microscopic balances.

Applications of the multiple gradient model are to processes with turbulent flow or to flow in highly complicated passages such as found in packed beds or porous media, process where the local detailed velocity field can not be either measured or calculated.

Maximum gradient description is more less detailed mode of multiple gradient description. In one sense this model may be considered to be just a simplified multiple gradient model in which the dispersion terms are avoided, and only one derivative is retained in the bulk flow terms. However, the model is employed so frequently that has set it aside as a separate category. Very often the scientist does not wish to attempt to analyze a process with the internal detail of the multiple gradient models. Additional simplifying assumptions are made, leading to mathematical equations that are easy to deal with and quite satisfactory for many purposes. In the maximum gradient model all dispersion is neglected, and only the largest, one-dimensional, component of the gradient of the dependent variable is retained in each equations.

For example, in the maximum gradient representation of a chemical reactor or gas absorber, only concentration gradients in the axial direction caused by the bulk flow are considered, and all radial gradients, dispersion, and the like are ignored.

In the process systems engineering maximum gradient models are the models commonly treated for continuous processes. The maximum gradient model is often called the "plug flow" model in other engineering fields. The maximum gradient models are described by ordinary differential 
equations.

To analyze a phenomena in microscopic level or even the internal detail of the multiple- or maximum- gradient models very often is complex. Instead, the macroscopic description is written all detail inside the specified subsystem, and as a consequence no spatial gradients are involved in the mathematical statements.

In facts, only time remains as a differential independent variable in the general balancing. The dependent variables, such as concentration and temperature, are not functions of position and hence represent averages over the volume of the subsystem. This loss of details greatly simplifies the mathematical description, but accompanying the simplification is a loss of information concerning performance characteristics of the system.

For a process with clearly defined boundaries, the macroscopic models for mass, momentum and energy conservation are represented by ordinary differential equations.

Mass of component A balancing including interphases transfer state as:

$$
\frac{d}{d t} m_{A, t o t}=\Delta\left(\rho_{A} v S\right)+q^{m}+S_{r}
$$

where $m$ is mass, $\Delta\left(\rho_{A} v S\right)$ represents difference of the mass flow rate between cross section 1 and $2, q^{m}$ is mass flow rate with interphases transfer through the cross section boundaries different from 1 and 2 , and $S_{r}$ is generation term.

\section{Integrated Modeling Method}

First step in system integration represents selection of range, element or subsystems, and connecting common parameters.

$$
M_{i}=F\left(R_{i}, e_{i}, p_{i}, y_{i}\right)
$$

where $M$ model, $F$ is aim function, $R$ is range, $e$ is element, $p$ is parameter, $y$ is dependent variable.

The second step requires establishing aim function and range. The third step requires interpretations of elements, subsystems and systems and the corresponding connections determination. Structural and functional integration is achieved by synthesis of hierarchical levels. At this the following criterion must be satisfied:

$$
M_{i-1} \in M_{R}
$$

where $M$ model on observed level, and $R$ is region of the system.

This relation can formulate as the first principle of integration.

Let consider integration in a chemical process system modeling. The process denotes an actual series of operations or treatment of materials, while model represents a mathematical description of the real process. Process model development can be expressed as the following manner.
Mathematical description of a process of the chemical product scale can be represented by individual reaction rates and total reaction rate can be defined as:

Let an exothermic reaction occur in the liquid phase,

$$
a A+b B \rightarrow^{k} c C
$$

or in equivalent form

$$
v_{a} A+v_{b} B+v_{c} C=0
$$

where

$$
\begin{gathered}
v_{a}=-1, v_{a}=-b / a, v_{C}=c / a=1 \\
\frac{d c_{A}}{d t}=\frac{d c_{B}}{d t}=-k c_{A}{ }^{n_{A}} c_{B}^{n_{B}} \\
\frac{d c_{C}}{d t}=k c_{A}{ }^{n_{A}} c_{B}{ }^{n_{B}} \\
\left(v_{r}\right)=A_{0} e^{-E / R T} c_{A}{ }^{n_{A}} c_{B}^{n_{B}}
\end{gathered}
$$

where $c$ is concentration, $k$ is specific kinetic constant, $\mathrm{s}^{-1}$, $E$ is energy activation, $A_{0}$ is frequency factor, $v_{r}$ is reaction velocity, $n$ is reaction order.

Total material and energy flows:

$$
F_{i}-F_{0}=\frac{d V}{d t}
$$

$$
Q=U A_{H}\left(T-T_{j}\right)
$$

Component conservation:

$$
\begin{gathered}
F_{0} c_{A 0}-F c_{A}-V\left(v_{r}\right)=-\frac{d}{d t}\left(c_{A} V\right) \\
F_{0} c_{B 0}-F c_{B}-V\left(v_{r}\right)=-\frac{d}{d t}\left(c_{B} V\right) \\
V\left(v_{r}\right)-F c_{C}=\frac{d}{d t}\left(c_{C} V\right)
\end{gathered}
$$

Energy conservation:

$$
\begin{aligned}
& \rho c_{p}\left(F_{0 A} T_{0}+F_{0 B} T_{0}-F T\right)-\Delta H_{r}\left(V\left(v_{r}\right)\right)+ \\
& U A_{H}\left(T_{J}-T\right)=\rho C_{p} \frac{d}{d t}(V T) \\
& \rho_{J} F_{J} C_{p J}\left(T_{J 0}-T_{J}\right)+U A_{H}\left(T_{J}-T\right)= \\
& -\rho_{J} V_{J} C_{P J} \frac{d T_{J}}{d t}
\end{aligned}
$$

where $F_{i}$ inlet flow rate, $F_{o}$ is outlet flow rate, mole/s, $T$ is temperature, $\mathrm{K}, C_{P}$ is heat capacity, $\mathrm{J} /$ mole $\mathrm{K}, \Delta H_{r}$ is reaction heat, $\mathrm{J} / \mathrm{mol}, U$-overall heat transfer coefficient, 
$\mathrm{J} / \mathrm{m}^{2} \mathrm{sK}, A_{H}$-heat transfer area, $\mathrm{m}^{2}$.

A new approach in modeling and simulation is represented directly by the structure of the elementary transitions referring to the various phenomenological defined reactions.

Since, the process denotes an actual series of operations or treatment of materials, by changing of a operation or materials, changes process charactersitics with the aim seeking out optimal process and products quality, and over them is introduced innovation in the process, that enabling innovation technology development.

The knowledge based system which automated the process, enabling engineers to perform modeling and simulation study was built. Input component data base and experimental database was linked with routines for kinetic parameters determination [3]-[6]. Database of kinetic parameters was developed as a relational data base system which linked kinetic models and operation simulation by process models.

The models manager operations performs real time process operations on various levels. Model manager shows how do you seek out a new way to the operation life cycle model and how do you make process history. It provides rigorous on-line modeling tools for the process design and operation and raw material and energy minimization [2]. This simulation integrates data bases for component data, chemical reaction data, dynamic data and model parameters tables with structured knowledge representation subsystems. Process and product innovation have been developed[12]-15].

\section{Functional Modeling}

In functional approach system modeling generates procedural, or rule based from a goal based architecture which further supports the development of secondary goals.

Knowledge representation has always been central topic research identify there presentation scheme of logic, procedural representation, semantic networks, production systems, direct analogical representations, semantic primitives, and frames and scripts[1]. Logic is too powerful because the need to acquire knowledge automatically from teacher or environment and integrate it with what is already knows. A representation of facts or rules only becomes knowledge when used by a program to behave in a knowledgeable way.

Current process acquisition systems perform routine housekeeping, permit rote learning of explicitly presented facts, and are able to elicit from experts simple rules based on the attributes. Methods of concept learning may be able to overcome these imitations, although the present state of the art is primitive and suggests ideas rather than well developed algorithms for the knowledge acquisition tool box. Concept learning systems take examples and create general descriptions, often expressed as rules, which process systems need [2]-[5].

It many years since process modeling become an advanced tool for design work in many companies. For example, process plant model objectives include to provide a comprehensive report of materials and energy streams, determine the correlation between process units study the formation and separation of byproducts and impurities, support preventive maintain by tracking performance of key equipment over time and its relation to the buildup of impurities [6]-[9].

Impurities in raw materials, incomplete conversion, byproducts, catalysts, auxiliaries, and secondary reactions as well as process energy make production residues. To understand the reason for the formation of residues in the chemical production processes, and to understand the possibilities for modifying these processes, the general conditions for chemical reactions must be considered[1].

\subsection{The Main and Intermediate Products Modeling}

$$
\text { Raw materials } \rightarrow X+S_{1}+I_{1}
$$

2.Main product:

$$
\begin{gathered}
X \rightarrow P+S_{2}+I_{2} \\
\text { 3. } S_{2} \rightarrow X+S_{3}+I_{3}
\end{gathered}
$$

where $\mathrm{P}$ is the main product, $\mathrm{X}$ is an intermediate product, $\mathrm{S}$ is secondary products, and $I$ is designated impurities, each of which can include a variety of components such as $I_{1}, I_{2}, I_{3}$.

As an example, an equilibrium reaction is considered in which a starting material $A$ reacts with a reaction partner $B$ to give a product $P$.

$$
A+B+N \stackrel{M, C . H . E}{\rightarrow} P+P^{\prime}+P^{\prime \prime}+N^{\prime}
$$

where $A$ is the starting material, $B$ the reaction partner, $N$ secondary constituent of $A$ and $B, N^{\prime}$ is the reacted secondary constituent, $M$ is the reaction medium, $C$ is a catalyst, $H$ is an auxiliary, $E$ is the energy, $P$ is the product and $P^{\prime}, P^{\prime \prime}$ a side product or byproducts.

The term residues denotes all components that take part in the reaction and do not give the desired end product $P$.

\subsection{Support in the Process System Modeling}

As a step toward a complete knowledge representation scheme for modeling support, it has combined the decomposition, taxonomic and coupling relationships in a knowledge representation scheme called the system entity structure. The elements represented are motivated, on the one hand, by system theory concepts of decomposition, how a system is hierarchically broken down into components and coupling how these components may be interconnected to reconstitute the original system. On the other hand, systems theory has not focused on taxonomic relations, as represented for example in frame hierarchy knowledge representation schemes.

The segregation of the databases and knowledge base systems in the process system allows us to organize the different models and domain expertise efficiently because each of these components can be designed and modified 
separately. The system approach permits the evaluation of feasibility and global plant integration, always for a predicted behavior of the process dynamic systems.

Simulations of the chemical process systems are more effective because of power to make possibilities to knowledge keep on with changeable technologies, products and the other sources. In the other hand simulation by models is increasing relation teacher and student, and stay with student during whole course and further, makes shorter training time and increasing learned skills level. An approach refers to the technique which predicts the possible qualitative behaviors of a system on the basis of the model comprising the predefined transfer parameters and constraint predicates. The quantitative approach to modeling and inference involve computing the path coefficients between the variables and using the resulting equation to predict the change in the cause. Although the quantitative approach has proven very useful for dealing with many real-world problems, it is neither sufficient nor necessary under some circumstances. Furthermore, because in reality, there may not exist enough quantitative knowledge to permit full quantitative modeling, abstract qualitative models are worthwhile to explore.

In structural domains, each example comprises a scene containing several objects, expressed in predicate logic. Part of the problem in matching a scene with a structural description is determining an appropriate mapping between objects in the scene and those specified in the description. This mapping will have different interpretations depending on whether the scene is to compromise or merely to contain the desired object. Several theoretical results indicate that, even in the simplest cases, extreme computational complexity can be involved in working with version spaces of structural objects.

In the case of a tree, the root specifies an attributte to be selected and tested first, then depending on its value subordinate nodes dictate tests on further attributes. The leaves are marked to show the classifications of the objects they represent. For two class problems these are simply "positive«or »negative«, but it easy to distinguish more than two classes. The algorithm uses an information theoretic heuristic to find a simple tree which classifies all examples given [3].

When presented with noisy data, it construct huge decision trees which reflect the detail of every example seen. In the case of production rules, the training set is used to construct a set of rules which can be interpreted by an expert system in standard forward or backward chaining manner

An extreme case of nonhierarhical representation is where each object is represented by an attribute vector as a semantic network. Real world knowledge bases are likely to fall between the extremes of hierarhical and completely flat representations. It has been extended to deal properly with such situations by thoroughly investigating competing hypothesis at each stage. If such an object does not exist, the alternative hypotheses are investigated to discover the most general valid desription.

It is often argued that an explanation driven process is essential for reasonable generalization. Correlation derived purely from empirical observations are much less convincing than a theory which explains them. The earliest example of expanation based learning, learns problem solving heuristics in the domain of symbolic integration. It usualy presumes the existance of a strong domain theory in which proofs can be constructed that show why a particular example is valid but some experiments have been reported with a kind of explanation based learning supported by very weak theory in the realm of physical causality for evereyday events.

An extreme case of nonhierarhical representation is where each object is represented by an attribute vector as a semantic network. Real world knowledge bases are likely to fall between the extremes of hierarhical and completely flat representations. It has been extended to deal properly with such situations by thoroughly investigating competing hypothesis at each stage. If such an object does not exist, the alternative hypotheses are investigated to discover the most general valid desription.

Process plant model should mirror the behavior of a complex plant subject to constraints in feedstock, products, equipment capacities, operational parameters, and utilities consumption. It's objectives include to:

- Provide comprehensive report of material and energy streams;

- Determine the correlation between the chemical reactors and separation systems;

- Study the formation and separation of byproducts and impurities;

- Improve in robustness to operation;

- Asses how to eliminate wastes and prevent environment pollution;

- Evaluate flexibility to changes in feedstock or products;

- Validate process instrumentation and enhance process control;

- Update process documentation and prepare

- Future investment, and

- Optimize the economic performance of the process.

Modeling and knowledge based simulation can integrate steps required to chemical process development. The general framework presented here on the model development side, the issues of knowledge representatioon in the form of systematic composition, ontology, and quantity representation was involved. On the model analysis side issues involving the automatic evaluation and presentation of simulation results.

On the most widely used forms of simulation is that for operator training. So far, operator training simulators have tended to use greatly simplified models in order to ensure real time performance and most effort has been invested in the development of user interface. A further ascpect of the extended application of simulation for operator assistance could well be achieved in conjunction with others systems.

The steady state model, which is simpler to build, and has a wide variety of applications in its own right, it can be used directly in revamping and a wide variety of other engineering projects. Dynamic simulation is a process engineering tool that predicts how process and its controls respond to various upsets as a function of time. 
In design, attention focuses on the main elements of material and heat balances, on equipment investment, and more generally, on process economics. While a deeper systems analysis of the plant would be worthwhile, considering that the basic design could be responsible for more than $80 \%$ of the cost of investment and operation, a detailed simulation and constrained, however, by the project schedule and lack of data. In design phase, flowsheetings are derived.

In fact, in flowsheeting only need an accurate description of the transformation linking the input the output of the process system.

In operation, attention centers mainly on product flow rate and specifications, but also plant troubleshooting, controllability, and maintenance. The performance of reactors and separators systems impose the rules of the game. They are independent and time variable to some extent. Only a detailed plant simulation enables an understanding of these interdependencies process knowledge of a detailed material and energy balance is by far more important in operations than in design. Even the flow rates of trace impurities are relevant, because they may impact equipment maintenance and environment protection [10]. The material and energy balance as well as operational characteristics of a plant are highly interconnected, and well suited for a system analysis. The life cycle concept may lead to a reliable and maintainable tool.

\section{Results and Discussion}

Because summary of models is just that, a summary, it may be necessary to consult other references to certain topics not previously encountered. More complete treatment of the development of microscopic and macroscopic models can be provided.

Simulation by molecular-, micro-, and macro-models carried out for various operation regions and object functions in the aim of the product quality innovation and environmental conditions improving. With data bases management and mathematical modeling of the process operation provides operation support system.

Functional modeling in the process systems analysis was considered.

Simulation in data base and knowledge base environment leads to intelligent model development. Process sensitivity analysis can be performed by dynamic model for various conditions.

\section{Conclusion}

In this paper theory of modeling was studied. The basic principles and methods were derived. Integrated modeling method was examined. The molecular modeling method was considered. Fundamentals of the microscopic to the macroscopic models were studied.

Functional modeling approach was considered. The process plant operation models were discussed.

The results of this paper have shown power of the system modeling method.

\section{Notation}

$\mathrm{A}_{\mathrm{H}}$-heat transfer area, $\mathrm{m}^{2}$

$A_{0}$ - frequency factor

$B$-algorithm

$c$-concentration

$D_{e f}$-diffusion coefficient, $\mathrm{m}^{2} / \mathrm{s}$

$E$ - energy activation, $\mathrm{J} / \mathrm{mol}$

$F$ - aimed function

$F_{i}, F_{o}$ - inlet and outlet flow rate, respectively, mole/s

$k$ - specific kinetic constant, $\mathrm{s}^{-1}$

$l$-variant

$m$ - mass

$M_{j}^{S}$ - system model

$n$ - reaction order

$q^{m}$ - mass flow rate with interphases transfer

T-temperature

U-overall heat transfer coefficient, $\mathrm{J} / \mathrm{m}^{2} \mathrm{sK}$

$v_{r}$-reaction velocity

$v_{x}, v_{y}, v_{z}$-spatial velocities

$S$-surface, $\mathrm{m}^{2}$

$S_{r}$ - generation term

$x$ - independent variable

$z$ - parameter

$y$ - dependent variable

Greek symbols

$\Delta \mathrm{Hr}$-reaction heat, $\mathrm{J} / \mathrm{mol}$

$\mu_{j k}$ - average deviation

$\rho_{A}$-density

Index

$A$-component A

$j$-jacket

$H$-heat, enthalpy

\section{References}

[1] T. Mosorinac, J. Savkovic-Stevanovic A multistage, multiphase and multicomponent process system modeling, Inter. J. of Mathematical Models and Methods in Applied Science, Issue 1, vol.5, 2011,pp.115-125.

[2] J. Savkovic-Stevanovic, Process modeling and simulation, Faculty of Technology and Metallurgy, Belgrade University, Belgrade, Yugoslavia,1995.

[3] T. Mosorinac, Chemical product characterization, MCBC2012Mathematics and Computers in Biology and Chemistry, Iashi, Romania, 13-15 June, 2013.

[4] J.Djurovic, An inverse control of extraction column, Inter. J. of Mathematical Models and Methods in Applied Science, Issue 1, vol.5, 2011,pp.67-76.

[5] J. Savkovic-Stevanovic, T. Mosorinac, S. Krstic, R. Beric, Computer aided operation and design of the surfactants production, Computer Aided Process Engineering -17, Vol.24, Eds. V. Plesu P. S. Agachi, Elsevier Science, 195-200, 2007. 
[6] H.M.Blalock,,Jr.., Causal Inferences in Non Experimental Research, The Norton Library, New York,1964.

[7] J.Savkovic-Stevanovic, A system for discovering developing, Comput. Ecol. Eng., vol.5, No.1, 2009,7-13.

[8] J. Savkovic-Stevanovic, Mathematical modeling innovation, 15th WSEAS International Conference on Mathematical and Computational Methods in Science and Engineering (MACMESE '13) Kuala Lumpur, Malaysia, April 2-4, 2013.

[9] J. Savkovic-Stevanovic, M. Ivanovic, R. Beric, V. Manjencic, Dynamic of the laurconium-chloride production, The 4th European Congress of Chemical Engineering, Granada, Spain 21-25 Sept.,2003.

[10] J. Savkovic-Stevanovic, M. Misic-Vukovic, G. Boncic-Caricic B. Trisovic, S. Jezdic, Reaction distillation with ions exchangers, Sep. Sci. Techn. 27, (1992)613-630.

[11] J. Savkovic-Stevanovic, M. Ivanovic, R. Beric, V. Manjencic, A surfactant formation investigation, Comput. Ecol.Eng.vol.1 (2005)129-135.

[12] T. Mosorinac, J. Savkovic-Stevanovic, M. Ivanovic-Knezevic, S. Pejanovic, M. Perunicic. N. Boskovic-Vragolovic, Modelling of the substance transfer in the gas -liquid system with chemical reaction, Petroleum \& Coal, vol.54 (3) 278-286, 2012, ISSN 1337-7027.

[13] J. Savkovic-Stevanovic, Fuzzy logic control system modelling, International Journal of Mathematical Models and Methods in Applied Sciences,vol.3,327-334,2009, ISSN 1991-8763.

[14] J. Savkovic-Stevanovic and J. Djurovic, Process systems modeling and simulation, Process systems in Chemical Engineering, Organizer: M. Milivojević, The 2014 Chemical Engineering and Materials, Venece, Italy, March 15-17,2014.

[15] J. Savkovic-Stevanovic, R. Berić, L. Filipović-Petrović, S. Veličković, Knowledge based reaction operation, Comput. Ecol.Eng.,7(1) 23-28, 2011, ISSN 1452-0729.

[16] J. Savkovic-Stevanovic, T. N. Mosorinac, S. B. Krstic, R. D. Beric, L. E. Flipovic-Petrovic and M. D. Milivojevic, Process plant model for design and optimization, Comput. Ecol. Eng. vol.6(1)(2010)1-9, ISSN 1452-0729.

[17] J. Savkovic-Stevanovic, S. Krstic, M. Milivojevic, M. Perunicic, Process plant knowledge based simulation and design, Chapter in Computer Aided Process Engineering -18 ,Vol.25,Eds. B. Braunschweig, and X. Joula, Elsevier Science, p.289-294, 2008. 